\title{
Significant Spot Detection of Small Target Image in Natural Scene with Variable Neighborhood Search
}

\author{
Li Xiang*
}

Lingnan Normal University, Zhanjiang, 524028, China

\begin{abstract}
Through the bright spot detection of small target image in natural scene, which is capable of appealing people's visual attention for rapid detection and identification of targets. Notable highlights model of small target image in natural scenes is prone to bring visual error with the difference between centre and surrounding of the background image, and visual saliency detection is ineffective. Considering the differences between local pixel characteristics and features of adjacent pixels, variable neighborhood search for local significance of central peripheral difference is taken, an improved significant spot detection algorithm of small target image in natural scene is proposed, constructing NSDFB frequency domain decomposition structure of significant highlight in small target image. Based on multi-scale DoG filter overlay, the calculation of the rare degree for the overall characteristics of target in the image can also measure the significance of the target, and a variable neighborhood search method is proposed to achieve improved spot detection model. The experimental results show that, the algorithm can detect bright spot of small targets in natural scene with good performance, and it has higher application value in the fields of small target recognition and multimedia information feature detection.
\end{abstract}

Keywords: Detection, natural scene, significant spot, small target image,

\section{INTRODUCTION}

With a variety means of advanced data processing are gradually got wide applications, image processing technology has been all-round developed and progressed as a modernized means of information acquisition and processing in the background of rapid development of computer technology. The processing technology of digital image concentrates on dealing with large amounts of visual input information quickly, extracting and identifying targets efficiently, meanwhile, including image edge detection and image fusion technology [1]. In different areas, depending on the image content, you can use modern computer technology to achieve intelligent image recognition technology. Today's digital image processing technology, has already been widely used in the fields of medical imaging, space exploration, investigative experiment and news media production [2]. In the natural scene, small target images are visible everywhere, and the detection and identification of small targets is mainly to detect significance of model on interest highlights in human visual scene, achieving important information extraction and fast processing of massive image data, then used in target image processing, industrial fault detection, and military targets identification.

Traditional methods on significant bright spot detection of small target image in natural scene can mainly be divided into edge-blending method and spectral analysis highlights detection algorithm. Between them, edge-blending method is used to detect highlights by extracting significant edge features, and determine what details people interest in under visual scene; and the latter carries out classification assignments and multiscale amount of informationa calculation according to characteristic map to get the results of spectral analysis of the small target image, realizing detection of bright spot model. Literature [3] presents a new method of combining wavelet transform domain Canny factor with the small target image edge-blending in natural scenes, doing three layer wavelet decomposition for blur edge of small target image, and the high frequency components and low frequency components are weighted average fusion, then effectively improves the fuzzy target image fusion to achieve detection of significant highlights, but the description of reconstructed image effects after edge-blending process and evaluation mechanism is not enough. Literature [4] proposed an improved Markov random field small target image edge processing method in natural scene, and the Markov random field model is introduced into the high-resolution SAR segmentation and fusion process of image edge, showing the feasibility of the target image processing effects. Literature [5] proposed an algorithm on detection of significant highlights based on wavelet analysis, using frequency-domain feature fusion rules, fractal testing the edge information after wavelet decomposition, which is feasible in a degree. However, the significant highlight model of small target image is prone to visual error with the central periphery difference at the background image in the natural scene, which results in ineffective visual saliency detection.

Aiming at this problem, the article has improved the traditional saliency detection methods of small target image in natural scene, combining local contrast with the global rarity 
operation, variable neighborhood searching for the central periphery difference, and studies the significant highlights detection of small targets in natural scene, then demonstrates the effectiveness of the improved algorithm.

\section{MODEL OF SIGNIFICANT HIGHLIGHTS DETEC- TION AND PROBLEM DESCRIPTION}

\subsection{Model and Random Neighborhood Design}

Traditional significant highlights detection of small target image in natural scene has lower resolution [6-8]. Due to RARE model having high-resolution edge information extraction and integration of small target image highlights at similarity domain of the same scale and multi-scale statistical correlation, this paper chooses multiscale variable neighborhood search random field to construct the framework of edge significant highlights detection of the target image, calculates the feature rarity based on the method of overall rarity, and the model incorporates the prior distribution model of the edge of target image and partial similarity neighborhood information of pixel, and the edge fusion of small target image in natural scene can be achieved by Bayesian estimation methods. Protruding edge and global rarity, decompose the bright spot region of small target image edge into two-dimensional network point set, expressed as:

$$
S=\{s=(x, y) \mid 1 \leq i \leq M, 1 \leq j \leq N\}
$$

Where in the area size of significant highlights in target image is $M \times N, M$ and $N$ corresponds to the and length and width of small target image edge. In the image, the visual significance of the target depends on the degree of difference between target and the background, so edge of the small image can be mapped to a two-dimensional grid, named $S$, which makes the color characteristics of homogeneous region to achieve pixel tags rarefaction in the whole scene, decomposed as:

$$
X=\left\{X_{1}, X_{2}, \ldots, X_{m}\right\}
$$

Calculating overall rarity of the feature, the system of random field neighborhood with small target image in natural scene is obtained:

$$
\begin{aligned}
\operatorname{DoG}(x, y) & =\frac{1}{2 \pi}\left[\frac{1}{\sigma_{1}^{2}} e^{1 \frac{\left(x^{2}+y^{2}\right)}{2 \sigma_{1}^{2}}}-\frac{1}{\sigma_{2}^{2}} e^{1 \frac{\left(x^{2}+y^{2}\right)}{2 \sigma_{2}^{2}}}\right] \\
& =G\left(x, y, \sigma_{1}\right)-G\left(x, y, \sigma_{2}\right)
\end{aligned}
$$

In the formula above, $\sigma_{1}$ and $\sigma_{2}$ represent the Gaussian standard deviation, respectively, also refer to as scale of Gaussian function, $G\left(x, y, \sigma_{1}\right)$ and $G\left(x, y, \sigma_{2}\right)$ are results of the original image after Gaussian convolution. Initially described in the above model, through constructing the estimated relationship between a priori distribution and a posteriori random probability, determine edge pixel fusion point feature of the small target image in natural scene, and build potential function model of information metric similarity neighborhood, using inverse transform of the potential function to obtain multi-scale local significance features.

\subsection{Significant Highlights Computational Model and Directional Filter Bank Design}

Considering the differences between local pixel characteristics and adjacent pixels features, build a significant highlight computational model, which is based on directional filter bank design, using PCA to reduce dimensions of the small target image in natural scene, by constructing a translation invariant direction filter group for downsampling and upsampling operations, due to the rapid changes of image features in the natural scene can attract human's attention, especially when there is no great change in color and grayscale of the edge region and the peripheral region, computing the local contrast of the image can outstand the significant goal of the image. This paper designs a directional filter bank to achieve this calculation process, using multi DoG filter overlay to get a 4-channel NSDFB decomposition structure shown in Fig. (1), the first stage is a two-channel sector filter bank, the filter of the second stage $U_{0}\left(z^{Q}\right)$ and $U_{1}\left(z^{Q}\right)$, through the first stage sector filter $U_{0}(z)$ and $U_{1}(z)$ , then sampled multiscale DoG filter overlay, leaving only the largest and smallest items, using the saliency map based on local contrast can extract the local place with strong contrast, and achieve detection of the bright spots model. In Fig. (1), supported by a square-shaped frequency domain, by calculating the rarity of the overall target characteristics in the image can also be used to measure the significance of target, using a combined two-stage filter to achieved frequency resolution of four directions, significant highlights of small target image corresponding NSDFB decomposition structure in the frequency domain is shown in Fig. (2).

\section{THE IMPROVED ALGORITHM DESCRIPTION}

The model above is mainly to compute pixel its local neighborhood pixels, under the framework of fredom field of significant highlights in multiscale small target image, traditional algorithms can not effectively display the highlights appeared in the whole figure, based on the maximum likelihood criterion, this article will introduce in the information measurement factor for optimization guidance to edge fusion rules of small target image. Using variable neighborhood search method to improve the model of significant highlights detection, the improved algorithm is described as:

The visual significant of target depends on the difference between target and its background, using the Gaussian filter function $G(x, y)$ to convolve processing the edge of the target image $f(x, y)$, assuming a grayscale image $I(x, y)$ whose domain is $\Omega$ is divided into two non-overlapping regions $R_{1}$ and $R_{2}$ by a profile curve $C$, adopt DoG filter to extract the mutation information of local area: 


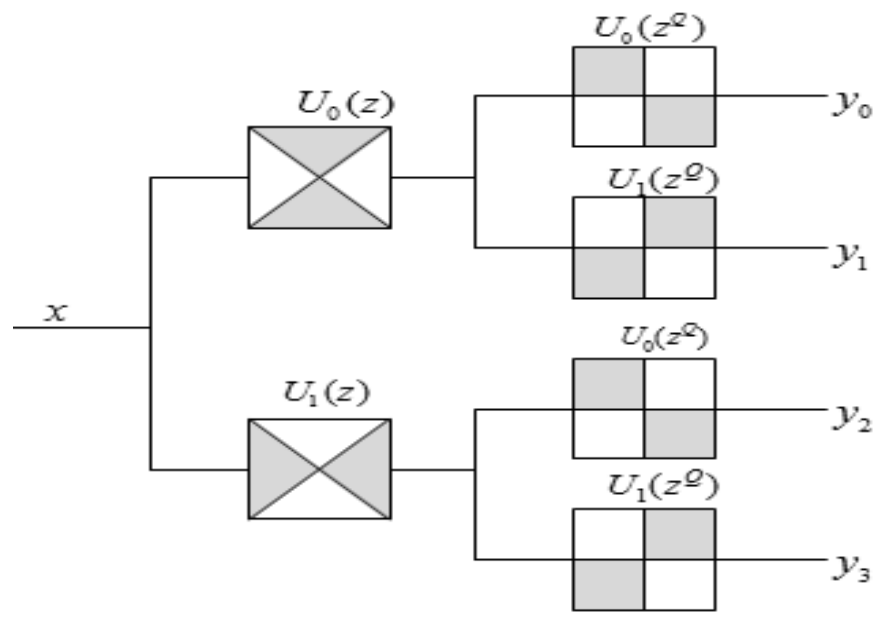

Fig. (1). Four channel NSDFB decomposition structure.

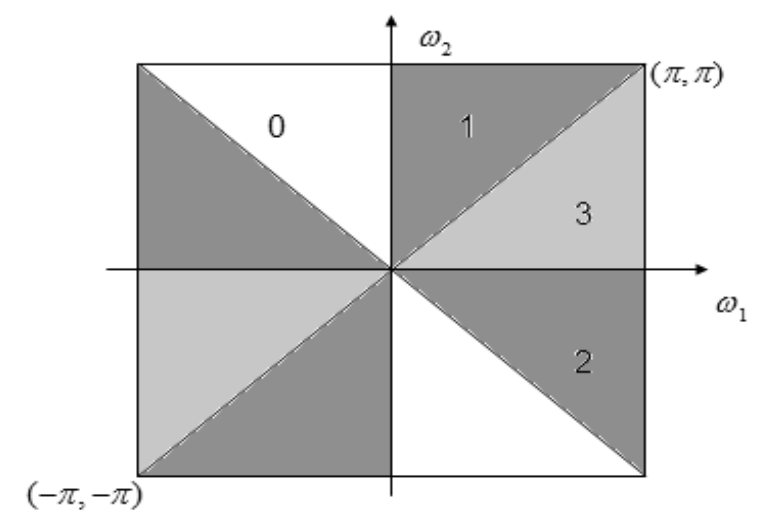

Fig. (2). Target image significantly highlight NSDFB domain decomposition structure.

$$
\begin{aligned}
E^{c v}\left(c_{1}, c_{2}\right) & =\mu \cdot \operatorname{Length}(C) \\
& +v \cdot \operatorname{Area}(\operatorname{inside}(C)) \\
& +\lambda_{1} \int_{\text {inside }(C)}\left|I-c_{1}\right|^{2} d x d y \\
& +\lambda_{2} \int_{\text {outside }(C)}\left|I-c_{2}\right|^{2} d x d y
\end{aligned}
$$

Among them, $c_{1}$ and $c_{2}$ represent the approximate average gray of the target area and the background area, respectively, an obvious global rarity target edge is formed after DoG filter overlay, and Length $(C)$ can be obtained using sparse coding method, which represents the length of the contour, Area(inside $(C)$ ) represents the vector radius of multi-scale feature outline curve, $\mu 、 v 、 \lambda_{1}$ and $\lambda_{2}$ represents the proportion of each energy item weighting coefficients in the whole scene, which all are greater than the constant 0 .

Estimating the edge energy feature of target image by Bayesian estimation method, using convolution integral to obtain a smooth blur aerial target image edge information, firstly, convolve with different scales of Gaussian kernel functions to obtain the statistical probability of characteristics and variance:

$$
\begin{aligned}
& I_{i f}(x, y)=I * G\left(x, y, \sigma_{i}\right) \\
& I_{i v}(x, y)=I * \operatorname{stdfilt}\left(x, y, w_{i}\right) \\
& S_{g i f}(x, y)=-\log \left(P_{i f}(x, y)\right) \\
& S_{g i v}(x, y)=-\log \left(P_{i v}(x, y)\right) \\
& S_{g i}(x, y)=S_{g i f}(x, y)+S_{g i v}(x, y)
\end{aligned}
$$

The equations above measure the overall significance of the pixel through statistical probability of feature and variance, in which $G\left(x, y, \sigma_{i}\right)$ represents a single scale Gaussian kernel function, $\sigma_{i}$ represents the calculating informationa after after with Gaussian kernel function, $w_{i}$ is the weight of Gaussian window function inside the homogeneous region of the target, namely, a scale of calculating the variance, 
Table 1. Parameter setting.

\begin{tabular}{|c|c|c|c|c|c|}
\hline Window Size & $\mathbf{3 * 3}$ & $\mathbf{5 * 5}$ & $\mathbf{9 * 9}$ & $\mathbf{1 7 * 1 7}$ & $\mathbf{3 3} \mathbf{3 3}$ \\
\hline \hline AUC & 0.236 & 0.362 & 0.897 & 0.991 & 0.963 \\
\hline
\end{tabular}

* stands for convolution, $I_{i}(x, y)$ is the information of different characteristics and variance, $P(x, y)_{i v}$ and $P(x, y)_{i f}$ are the probability that a pixel $(x, y)$ which appears in are overall characteristics of the target, respectively, $S_{g i v}$ and $S_{\text {gif }}$ are the significance images of characteristic variance and characteristic information when the image features change rapidly in the scene, $S_{g i}$ is the difference between the integrity of target and the significant information of homogeneity region.

Owing to the visual error traditional methods produce between significant highlights model for small target image and the central periphery difference of the background image in natural scene, the visual saliency detection is ineffective. The article adopts variable neighborhood search methods, introducing maximum likelihood threshold to control edge texture features of singlel-line, at the same time, caculating noise suppression threshold to achieve dual-threshold control, then effectively suppressing noise to maintain a good feature of integration information. Based on the MumfordShah model of traditional methods, using the local gray information of the image, it can be very good to overcome gray inhomogeneity, and the significant figure based on local contrast can extract the place with a strong local contrast, showing prominent edge feature, the level set function of homogeneous region in small target image is expressed as:

$C=\{(x, y) \in \Omega: \phi(x, y)=0\}$

Searching of variable neighborhood based on the changing information of local contrast, whereby the variable neighborhood search level set form based on central periphery difference of energy function is:

$$
\begin{aligned}
& E^{L B F}\left(\phi, f_{1}, f_{2}\right)= \\
& \quad \mu \int \frac{1}{2}(|\nabla \phi|-1)^{2} d x+v \cdot \text { Length }(C) \\
& \quad+\lambda_{1} \int\left[\int K_{\sigma}(x-y)\left|I-f_{1}(x)\right|^{2} H(\phi) d y\right] d x \\
& \quad+\lambda_{2} \int\left[\int K_{\sigma}(x-y)\left|I-f_{2}(x)\right|^{2}(1-H(\phi) d y)\right] d x
\end{aligned}
$$

Among them, $H(z)$ and $\delta(z)$ are Heaviside function and Dirac function, which can surface the global rarity of different pixels. Formula above has brought about improvement of using DoG filter to extract the mutation information of local area in traditional methods, effectively distinguishs between target and background, and avoids segmentation errors.
Finally, $P(X)$ represents the classification statistics prior probability of target image edge pixel, $P(Y)$ indicates the detection probability of small target image edges, usually a constant. According to statistical analysis, in the case of the target image edge for detection is given, the edge-blending information of image can be obtain on the basis of posterior probability distribution $P(Y \mid X)$, and the maximum probability density estimation $X$. In order to make the global significance with a certain scale invariance, using global significance operations to highlight the information of the features image, at the same time, combined with the highlight content of image at different angles and different scales, amalgamating the global significance calculated by multi-scale images to achieve significant highlights testing of variable neighborhood search, test results are as follows:

$S_{G}(x, y)=-\frac{1}{N} \sum_{i=1}^{N-1} S_{g i}(x, y)$

In which, $S_{g i}(x, y)$ represents the significance of pixel features at different scales. Combined with local comparison and global rarity operation, variable neighborhood search for the central periphery difference to achieve significance highlights detection of small target images in natural scene.

\section{SIMULATION RESULTS}

In the simulation experiments, through calculating the rarity of the whole characteristics of the target in the image to achieve significant highlights detection of a small target image in natural scene, in order to improve the speed of calculation, by calculating the information of different characteristics to obtain the global rarity of different pixels, while adding a certain amount of spatial information for simulation experiments, samples of the simulation tests are a variety of different target images in 1000 natural scenarios, the experimental hardware environment is described as: test platform for running is windows XP system, Pentium (R) 4 CPU 3.00 $\mathrm{GHz}$ and internal storage is $1 \mathrm{GHz}$. In order to achieve the performance comparison, based on the global rarity algorithm, combined with local comparative and global rarity of LG algorithm and RARE significant highlights detection algorithm to achieve performance comparison. In the simulation test, how to choose the size of the Gaussian kernel function has made many attempts to achieve optimal processing parameters, the parameters of neighborhood search based on local contrast window size are set in Table $\mathbf{1}$.

Using the method of calculating the bottom area of the ROC curve (AUC) to evaluate the effect significant 
Table 2. Variable neighborhood search detection combination mode.

\begin{tabular}{|c|c|c|c|c|c|c|}
\hline Combi-nation Mode & Max & L+3G & L+2G & L+G & 2L+G & 3L+G \\
\hline \hline AUC & 0.6395 & 0.6324 & 0.6322 & 0.3021 & 0.9632 \\
\hline
\end{tabular}
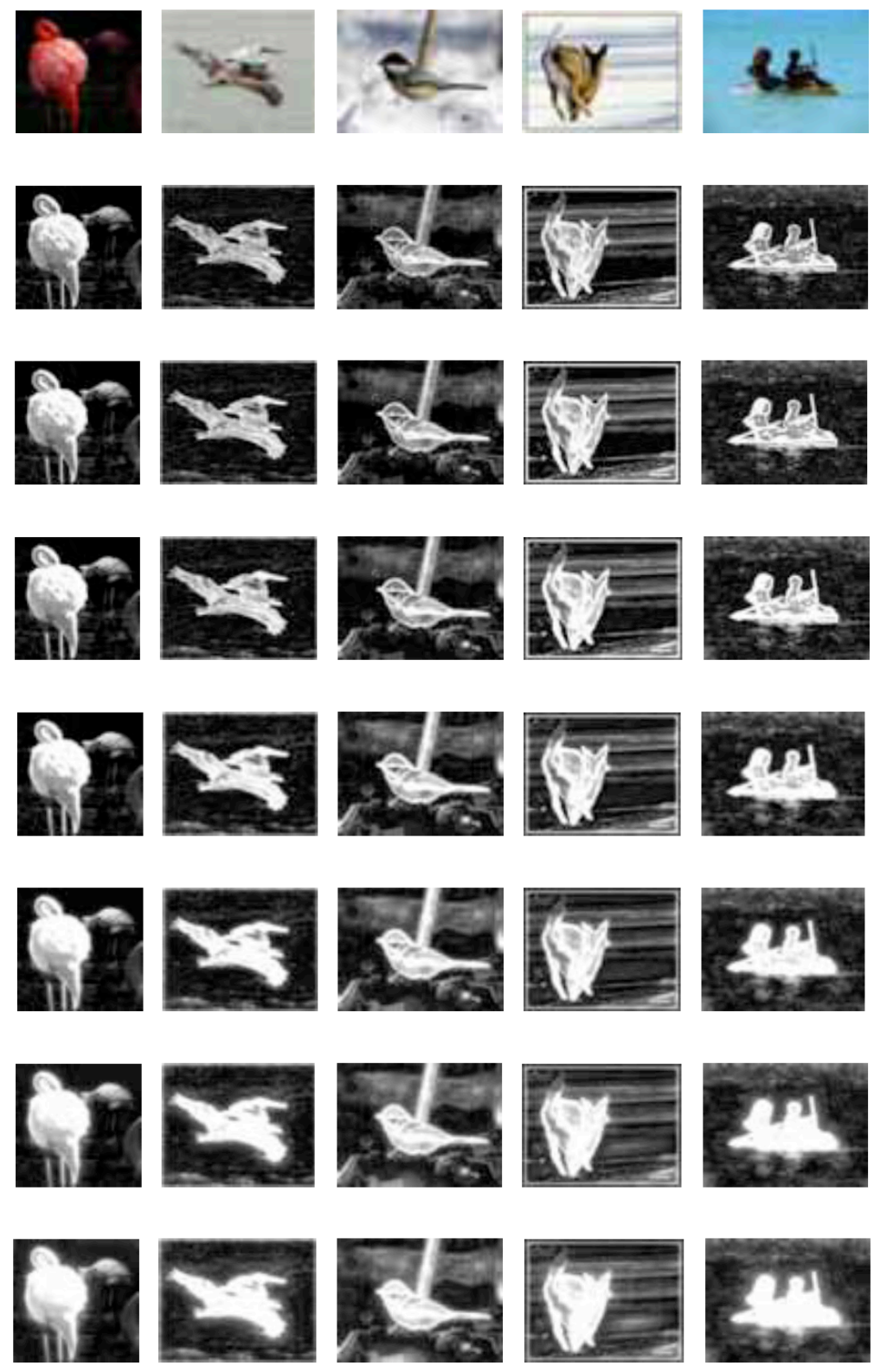

Fig. (3). Detection results of small image.

highlights detection of small target image in natural scenes, adopt multi-scale Gaussian filter to obtain a combination of global rarity to do variable neighborhood search, detection combination modes are shown in Table 2.

Based on the parameter setting and analysis above, when the coefficients of local contrast and the global rarity is $2: 1$ and a linear combination can be better performance of AUC to operate the simulation of significant highlights detection. The simulation results are shown in Fig. (3). In Fig. (3), the first one is the original image, followed by a single scale, two scales combined, three scales combined until seven scale integration, the performance of the significant highlights detection is good, the saliency map calculated by this method can better highlight significant targets. 


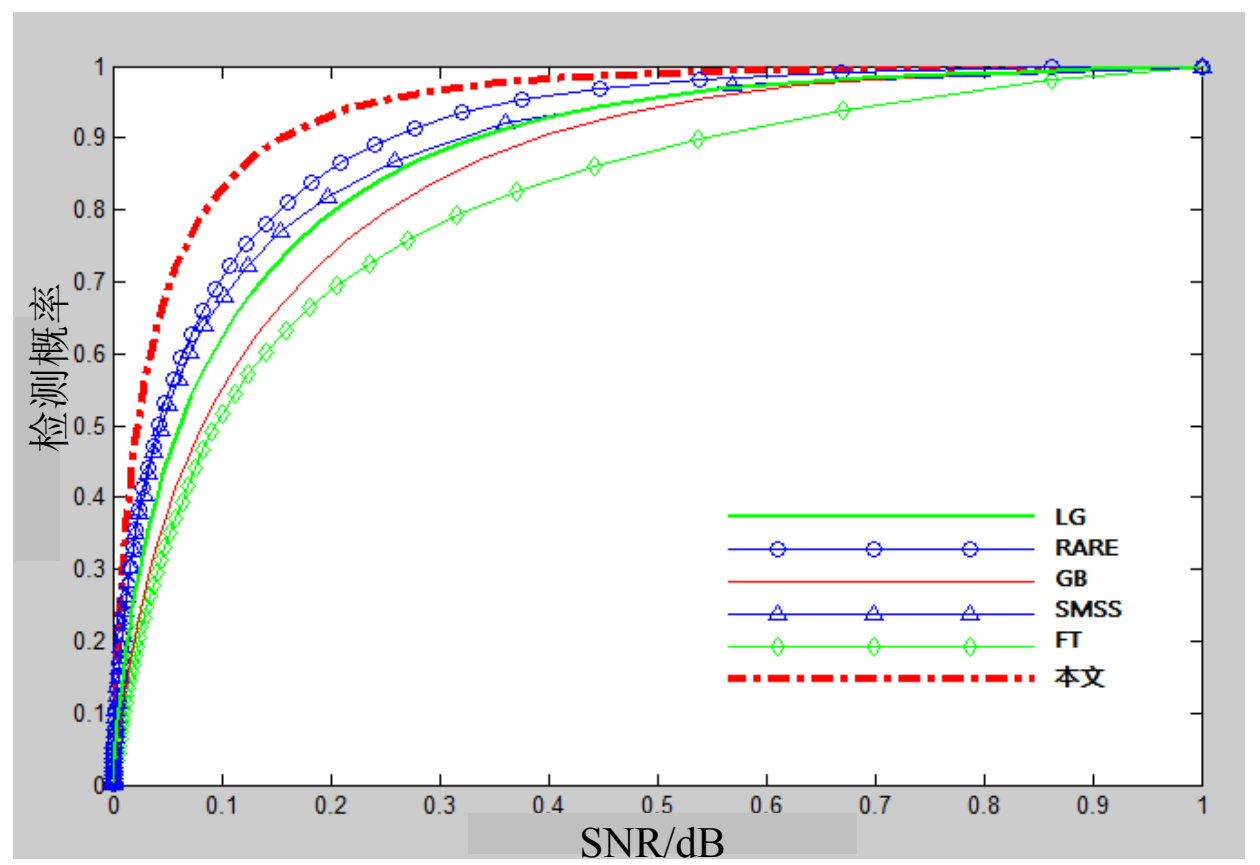

Fig. (4). Detection performance curve.

In order to analyze the test results quantitatively, based on Achanta database, using ROC figures analysis to calculate the detection performance, from Fig. (4) we can see, there is a high probability of detection using this algorithm and the performance is better than several other models. When the recall value is 1.0 , the precision of all methods is 0.2 , which indicates an average of $20 \%$ each figure in this database. The paper uses more color spaces as well as multiscale operations, which improves the detection performance of highlights models.

\section{CONCLUSION}

Owing to the visual error traditional methods produce between significant highlights model for small target image and the central periphery difference of the background image in natural scene, the visual saliency detection is ineffective. This paper considers the differences between local pixel features and adjacent pixels characteristics, variable neighborhood searching for local significance of the central periphery difference, an improved algorithm of significant highlights detection of small target images in natural scene is proposed, conducting random neighborhood system design, designing NSDFB frequency domain decomposition structure of significant highlights in small target images, adopting variable neighborhood search to achieve the improvement of significant highlights detection model, which achieves better performance of test results, improving the detection performance of the highlights model, and has a higher application value in the fields of small target recognition and multimedia information retrieval.

\section{ABOUT THE AUTHOR}

Li Xiang, born in 1972-02-16, MengZi, Yunnan province, China. Master graduate student degree. His research interests include algorithm research of communication, computer network technology, digital image processing algorithm.

\section{CONFLICT OF INTEREST}

The author confirms that this article content has no conflict of interest.

\section{ACKNOWLEDGEMENTS}

This work is supported by the Key Project of Science and Technology research project of Henan province:(Research of dynamic vehicle positioning system based on digital video image, No. 132102210423).

\section{REFERENCES}

[1] K. Lu, C. Li, and J. Zhao, "A fast sub-pixel image registration algorithm," Infrared Technology, vol. 35, no. 1, pp. 27-30, 2013.

[2] A. Toet, "Computational versus psychophysical bottom-up image saliency: a comparative evaluation study," IEEE Transactions on Pattern Analysis and Machine Intelligence, vol. 33, no. 11, pp. 2131-2146, 2011.

[3] Y. Guo, H.J. Yuan, and P. Wu, "Image saliency detection based on local and regional features," Acta Automatica Sinica, vol. 38, no. 9 , pp. 1513-1519, 2012.

[4] A. Borji, and L. Itti, "Exploiting local and global patch rarities for saliency detection," In: Proceedings of IEEE Conference on Computer Vision and Pattern Recognition, 2012, pp. 478-485. 
[5] N. Riche, M. Mancas, B. Gosselin, and T. Dutoit, "Rare: a new bottom-up saliency model," In: Proceedings of IEEE International Conference on Image Processing, 2012, pp. 641-644.

[6] Z. Li, M. Zhou, and G. Li, "The identification algorithm of similarity multiple targets based on wavelet transform," Bulletin of Science and Technology, vol. 29, no. 3, pp. 120-124, 2013.
[7] Z. Guo, Y. Zhang, and Z. Lin, "Multi-pose 3D facial landmark localization based on multi-model information," Chinese Journal of Computers, vol. 35, no. 1, pp. 163-170, 2012.

[8] S. Yang, Y. Qi, and X. Shen, "Rapid and automatic method for 3D scanned data registration," Journal of Software, vol. 21, no. 6, pp. 1438-1450, 2010.

Received: September 16, 2014

Revised: December 23, 2014

Accepted: December 31, 2014

(C) Li Xiang; Licensee Bentham Open.

This is an open access article licensed under the terms of the Creative Commons Attribution Non-Commercial License (http://creativecommons.org/licenses/by$\mathrm{nc} / 3.0 /$ ) which permits unrestricted, non-commercial use, distribution and reproduction in any medium, provided the work is properly cited. 